\title{
Small bowel haemorrhage due to cytomegalovirus vasculitis
}

\author{
J M Sackier, S B Kelly, D Clarke, A J Rees, C B Wood
}

\begin{abstract}
A case is described of a life threatening vasculitis of the small bowel leading to massive gastrointestinal haemorrhage which was apparently due to cytomegalovirus inclusion disease. Reactivation of cytomegalovirus probably followed the treatment of Wegener's granulomatosis with corticosteroids and azathioprine. This patient was treated successfully by surgical excision of the affected segment of ileum together with intravenous ganciclovir.
\end{abstract}

Cytomegalovirus inclusion disease most commonly affects infants under 2 years of age, and is rare in adults and older children.' Although a few cases of primary disease have been identified in adults without predisposing causes, it is found typically as an opportunistic infection associated with chronic debilitating diseases such as neoplasia (particularly leukaemia and lymphoma), haemodialysis, severe malnutrition, chronic infection, and treatment with corticosteroids, cytotoxic drugs, or ionising radiation. ${ }^{12}$ In adults the disseminated form has been known to affect the lungs, adrenals, liver, gastrointestinal tract, pancreas, spleen, and kidney and seems to be uniformly fatal. ${ }^{3}$ This case is unusual in that per oral colonoscopy was carried out at laparotomy, thus enabling accurate localisation of the site of bleeding in the ileum (as had been previously shown on angiography). This technique is rarely used; however, it proved to be successful in this case.

\section{Case report}

A 69 year old woman developed an influenza like illness, with haemoptysis, epistaxis, malaise, anorexia, and diarrhoea; two weeks later she developed a vasculitic rash. One month later, when still unwell, she consulted her doctor; her haemoglobin was $72 \mathrm{~g} / \mathrm{l}$ with an iron deficiency picture. On examination there was a pronounced vasculitic rash over her arms and legs, nail-bed splinter haemorrhages, ulcers on the hard palate, fine inspiratory crackles in the chest, and ankle oedema.

Chest $x$ ray films showed diffuse shadowing and urine analysis showed blood and protein. Urea was raised to $69 \mathrm{mmol} / \mathrm{l}$, creatinine to 1100 $\mu \mathrm{mol} / \mathrm{l}$, and clotting screen normal. A renal biopsy specimen showed focal necrotising glomerulonephritis. Wegener's granulomatosis was diagnosed and she was treated with peritoneal dialysis, blood transfusion, fresh frozen plasma, prednisolone $60 \mathrm{mg}$ once a day decreasing to $45 \mathrm{mg}$ after seven days, cyclophosphamide $100 \mathrm{mg}$ once a day, and seven four litre plasma

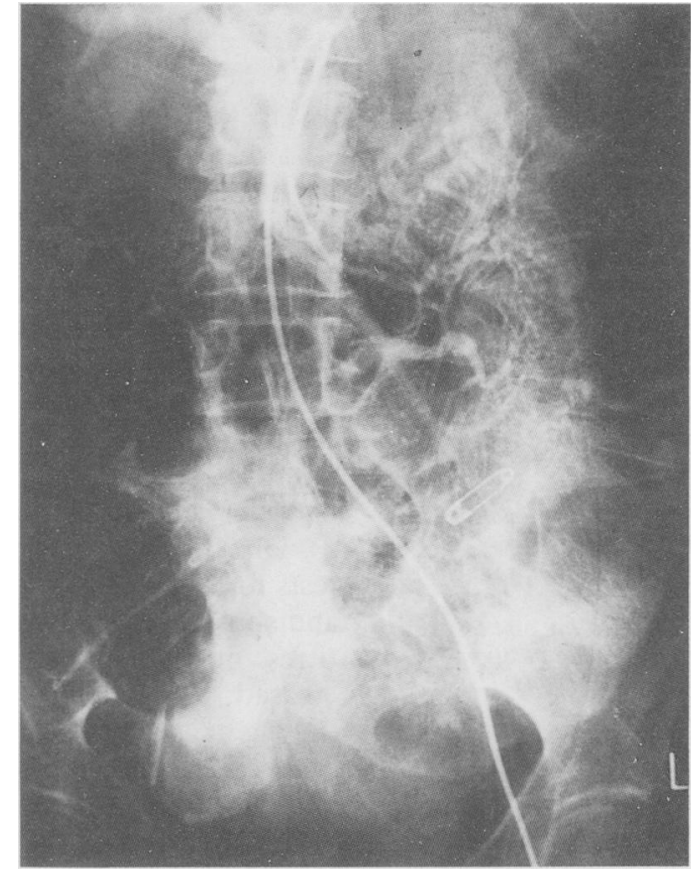

Figure 1: Superior mesenteric angiogram showing an obvious bleeding site in the terminal ileum.

exchanges for albumin. Her symptoms and renal failure responded. Four days later she developed melaena and became shocked. Endoscopy showed an old duodenal ulcer with no stigmata of active bleeding and she was treated with blood transfusion and $\mathrm{H}_{2}$ antagonists. She had further episodes of bleeding and angiography showed poor splanchnic vasculature on the superior mesenteric angiogram with an obvious bleeding site in the terminal ileum (Fig 1).

At laparotomy the small bowel was abnormal from the duodenojejunal flexure to the caecum with serosal telangiectasia, and the lumen was full of blood. Two palpably abnormal areas in the terminal ileum were excised. Blood was seen to come from proximal and distal ends in both diseased segments and in the resected bowel the mucosa was grossly ulcerated. The impression at operation was that there was disease of the whole of the small intestine and nothing would be gained by further resection.

For 24 hours the disease was quiescent and then she rebled. At laparotomy, per oral endoscopy with a colonoscope showed an actively bleeding vessel in the ileum distal to the most distal resection. This segment of ileum was removed and an end-to-end anastomosis performed. Postoperative progress was unremarkable.

Histology of the excised lesion showed a small artery in the base of an ulcer (Fig 2). Further histological examination showed inclusion bodies in both cytoplasm and nucleus of endo-

Renal Medicine, and

Pathology, Royal

Hospital, London

14 December 1990
} 


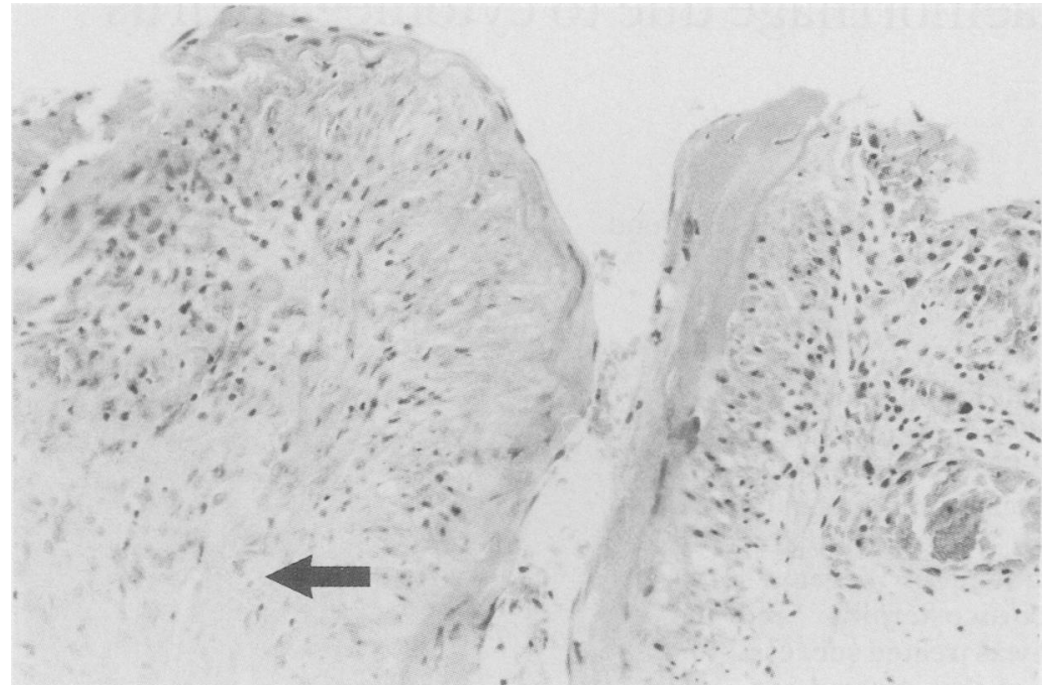

Figure 2: Base of the ulcer found at the second operation. Note the artery which was identified as the site of bleeding, and an endothelial cell in a capillary adjacent to it (arrow) containing an intranuclear inclusion body.

Haematoxylin and eosin, original magnification $\times 140$. thelial cells in the vessel wall (Fig 2) and electron microscopy showed these to be herpes type virus particles consistent with cytomegalovirus (Fig 3). Direct early antigen fluorescence focus assay tests for cytomegalovirus were positive in blood and sputum, it was grown in tissue culture, and titres of anti-cytomegalovirus antibodies increased. The patient was treated with ganciclovir (60 mg daily for 11 days), recovered completely, and is currently well.

\section{Discussion}

Cytomegalovirus infection is common, up to $80 \%$ of adults having acquired complement fixing antibodies to the virus by 35 years of age. ${ }^{3}$ Cytomegalovirus inclusion disease may exist in a localised or disseminated form in both adults and infants. The localised form of the disease in adults is usually limited to gastrointestinal ulcers or to the lungs. ${ }^{+}$Cytomegalovirus inclusions have been described with increasing frequency in a variety of gastrointestinal lesions, including ulcerative colitis, idiopathic steatorrhoea, afferent loop syndrome, and ulcerations of the oesophagus, stomach, small intestine, and rectum. ${ }^{2}$ The disseminated form in adults commonly occurs as a terminal complication of a severe debilitating disease. Its clinical features are poorly known, since they are usually obscured by the signs of the associated systemic disease.

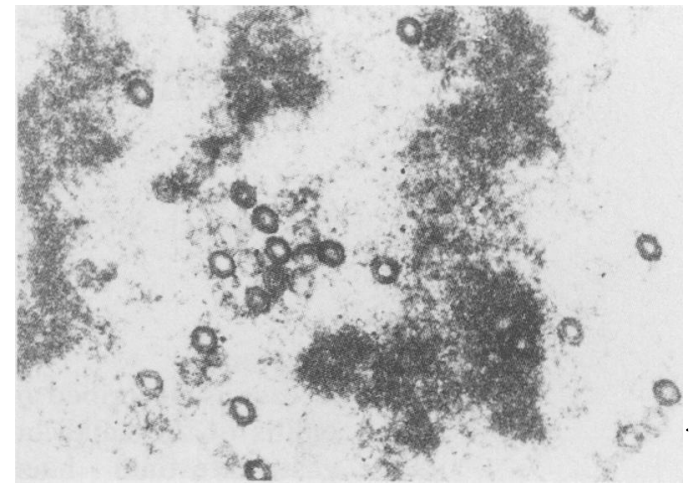

Figure 3: Electron micrograph of an intranuclear inclusion in an endothelial cell and the base of one of the ileal ulcers. Note the many herpes type viral particles. Original magnification $\times 21000$.

The mechanism by which this disease causes bleeding is largely due to viral localisation preferentially in vessel walls, where damage is caused by endothelial anoxia, ${ }^{5}$ leading to vessel wall damage. ${ }^{6}$ In addition, megakaryocyte production is decreased and disseminated intravascular coagulation may occur. ${ }^{7}$ Gastrointestinal bleeding has been well documented in other species, such as equine viral arteritis. ${ }^{8}$

Cytomegalovirus inclusion disease is a relatively frequent complication of immunosuppressive treatment for systemic vasculitides, such as Wegener's granulomatosis. These vasculitides can also affect the gastrointestinal tract to cause haemorrhage. Histological changes in the gut in our patient, however, were not compatible with Wegener's granulomatosis and it is likely that cytomegalovirus reactivation had occurred due to the corticosteroid and cytotoxic treatment of the primary disease.

1 Levine RS, Warner NE, Johnson CF. Cytomegalic inclusion disease in the gastrointestinal tract of adults. Ann Surg 1964; 159: $37-48$.

2 Goodman MD, Porter DD. Cytomegalovirus vasculitis with fatal colonic haemorrhage. Arch Pathol 1973; 96: 281-4.

3 Henson D. Cytomegalovirus inclusion bodies in the gastrointestinal tract. Arch Pathol 1972; 93: 477-82.

4 Wong TW, Warner NE. Cytomegalic inclusion disease in adults. Arch Pathol 1962; 74: 403-22.

5 Estes PC, Cheville NF. The ultrastructure of vascular lesions in equine viral arteritis. Am $\mathcal{F}$ Pathol 1970; 58: 235-40.

6 McKay DG, Margaretten W. Disseminated intravascular coagulation in virus diseases. Arch Intern Med 1967; 120: 129-52.

7 Klein R, Robboy SJ. Case records of the Massachusetts General Hospital, Case 7. N Englf Med 1973; 288: 363-7.

8 Jones TC, Doll ER, Bryans JT. The lesions of equine viral arteritis. Cornell Vet 1957; 47: 52-68. 